Pacific Journal of Mathematics

STARLIKE INTEGRAL OPERATORS 


\title{
STARLIKE INTEGRAL OPERATORS
}

\author{
Sanford S. Miller, Petru T. Mocanu, and Maxwell O. Reade
}

Let $S^{*}$ denote the set of all functions $f(z)=z+\cdots$ univalent and starlike in the unit disc $U$. The authors show that integral operators of the form

$$
\mathscr{I}(f) \equiv\left[\frac{\beta+\gamma}{z^{\gamma} \Phi(z)} \int_{0}^{z} f^{\alpha}(t) \varphi(t) t^{\grave{-}-1} d t\right]^{1 / \beta}=z+\cdots
$$

with suitable restrictions on the analytic functions $\Phi(z)$ and $\varphi(z)$, for suitable choices of the constants $\alpha, \beta, \gamma$, and $\delta$, transforms $S^{*}$ into $S^{*}$. With other restrictions on the parameters, the authors obtain transformations of the sets $K$, $S^{*} \times K, K \times K$ into $S^{*}$. Here $K$ denotes, as usual, the set of all $f(z)=z+\cdots$ univalent and convex in $U$.

1. Introduction. Let $S^{*}$ be the set of all functions $f(z)=z+\cdots$ analytic, univalent and starlike in the unit disc $U$, and let $S^{*}(\alpha)$ denote the subset of all $f \in S^{*}$ that satisfy $\operatorname{Re}\left[z f^{\prime}(z) / f(z)\right]>\alpha$ in $U$, $0 \leqq \alpha<1$. $S^{*}(\alpha)$ is the set of all (normalized) functions starlike of order $\alpha$ in $U$, and $S^{*}(0) \equiv S^{*}$. The set of all functions $f(z)=z+\cdots$ analytic, univalent and convex in $U$ is denoted by $K$, and the set of all $f \in K$ that satisfy $\operatorname{Re}\left[1+z f^{\prime \prime}(z) / f^{\prime}(z)\right]>\alpha$ in $U$ is denoted by $K(\alpha)$. The set $K(\alpha)$ consists of all functions convex of order $\alpha$ in $U$ and $K(0) \equiv K$. Moreover, it is well-known that $K \subset S^{*}(1 / 2) \subset S^{*}$, and it is known that $f \in K$ if and only if $\left(z f^{\prime}\right) \in S^{*}$.

Many authors have studied operators of the form

$$
\mathscr{S}(f) \equiv \frac{1+\gamma}{z^{\gamma}} \int_{0}^{z} f(t) t^{\gamma^{-1}} d t
$$

where $\gamma$ is a real constant and where $f$ belongs to some favored class of functions $[1,2,4,5,6,7]$; they have obtained simple results by certain direct methods. However by using a more general operator combined with a new method we obtain results that are both more general and sharper than those obtained heretofore. We also pose some questions that we believe are of sufficient interest to (attempt to) answer.

Our fundamental theorem is stated and proved in $\S 2$; in $\S 3$ we offer some applications of our fundamental theorem. Variations of our basic result that lead to transformations of $K, S^{*} \times K$, and $K \times K$ into $S^{*}$, are given in $\S \S 4,5$, and 6 , respectively.

2. Fundamental theorem. We need the following lemma [3]. 
Lemma A. Let $u$ and $v$ denote complex variables, $u=u_{1}+i u_{2}$, $v=v_{1}+i v_{2}$, and let $\Psi(u, v)$ be a complex-valued function that satisfies the following conditions. (a) $\Psi(u, v)$ is continuous in a domain $\mathscr{D} \subset C^{2}$. (b) $(1,0) \in \mathscr{D}$ and $\Psi(1,0)>0$. (c) $\operatorname{Re}\left(i u_{2}, v_{1}\right) \leqq 0$ whenever $\left(i u_{2}, v_{1}\right) \in \mathscr{D}$ and $v_{1} \leqq-\left(1+u_{2}^{2}\right) / 2$. If $p(z)=1+\cdots$ is a function that is analytic in $U$, such that $\left(p(z), z p^{\prime}(z)\right) \in \mathscr{D}$ and $\operatorname{Re} \Psi\left(p(z), z p^{\prime}(z)\right)>0$ hold for all $z \in U$, then $\operatorname{Re} p(z)>0$ in $U$.

The Lemma, which is not difficult to prove, permits us to generalize certain known results and it permits us to obtain sharper results as well. One example is the following theorem which generalizes known results $[1,2,4,5,6,7]$.

THEOREM 1. Let $\Phi(z)=1+\cdots$ and let $\phi(z)=1+\cdots$ be analytic functions defined in $U$, with the property $\varphi(z) \cdot \Phi(z) \neq 0$ there. Let $\alpha, \beta, \gamma$, and $\delta$ be real constants such that

$$
\begin{gathered}
\alpha \geqq 0, \beta>0, \\
\delta \geqq 0, \\
\alpha+\delta>0,
\end{gathered}
$$

and

$$
\alpha+\delta=\beta+\gamma .
$$

If there exists a nonnegative constant $J$ that satisfies

$$
\begin{gathered}
J \geqq \gamma+\operatorname{Re}\left[\frac{z \Phi^{\prime}(z)}{\Phi(z)}\right], \\
\beta+\gamma>J, \\
\delta+\operatorname{Re}\left[\frac{z \phi^{\prime}(z)}{\varphi(z)}\right] \geqq \max [0, J-\lambda(J)],
\end{gathered}
$$

where

$$
\lambda(J) \equiv \frac{1}{2} \max \left[\frac{\beta+\gamma-J}{J}, \frac{J}{\beta+\gamma-J}\right], \lambda(0)=0,
$$

and if $f \in S^{*}$, then there exists a function $F(z)=z+A_{2} z^{2}+\cdots, F \in S^{*}$, that satisfies the identity

$$
F(z) \equiv \mathscr{J}(f) \equiv\left[\frac{\beta+\gamma}{z^{\gamma} \Phi(z)} \int_{0}^{z} f^{\alpha}(t) \varphi(t) t^{\hat{o}-1} d t\right]^{1 / \beta}=z+\cdots
$$

In (9) all powers are principal ones.

Note. First we note that the conditions (1)-(7) are not all in- 
dependent ones; we have enumerated the items to which we refer explicitly in the text.

Proof. We shall assume the existence of the function $F(z)$, satisfying (9), and give a proof of the assertion $F \in S^{*}$ first. After this proof, we shall show that a $F(z)$ satisfying (9) does indeed exist.

From (9) we obtain, via a differentiation,

$$
\beta F^{\beta-1} F^{\prime} \boldsymbol{z}^{\gamma} \Phi(\boldsymbol{z})+\gamma \boldsymbol{z}^{\gamma-1} \Phi(\boldsymbol{z}) \boldsymbol{F}^{\beta}+\Phi^{\prime}(\boldsymbol{z}) \boldsymbol{F}^{\beta} \boldsymbol{z}^{\gamma} \equiv(\beta+\gamma) f^{\alpha} \varphi(\boldsymbol{z}) \boldsymbol{z}^{\hat{j}-1},
$$

so that if in this last equation we make the substitutions

$$
p(z) \equiv \frac{z F^{\prime}}{F}, \quad q(z) \equiv \frac{z \Phi^{\prime}(z)}{\Phi(z)}+\gamma,
$$

and then take another logarithmic derivative, we obtain

$$
(\beta p+q)+\frac{z\left(\beta p^{\prime}+q^{\prime}\right)}{(\beta p+q)}-\left(\frac{z \varphi^{\prime}}{\varphi}+\delta\right) \equiv \alpha z \frac{f^{\prime}}{f} .
$$

Since $f \in S^{*}$, the right-hand member of (11) has a positive real part in $U$. We wish to show that the function $p(z)$ has a positive real part in $U$ too. To that end, we introduce a new constant $k$,

$$
k \equiv \beta+\gamma-J,
$$

as well as the new function

$$
P(z) \equiv \frac{1}{k}[\beta p+q-J]=\frac{1}{k}\left[\beta \frac{z f^{\prime}}{f}+\frac{z \varphi^{\prime}}{\varphi}+\gamma-J\right],
$$

in order to write (11) in the form

$$
k P(z)+\frac{k z P^{\prime}(z)}{k P(z)+J}+\lambda(J)=\alpha z \frac{f^{\prime}(z)}{f(z)}+\left[\left(\delta+\frac{z \varphi^{\prime}(z)}{\varphi(z)}\right)-(J-\lambda(J))\right] .
$$

In order to use Lemma $A$, we must verify that the function

$$
\Psi(u, v) \equiv \lambda(J)+k u+\frac{k v}{k u+J}
$$

satisfies the hypothesis of that lemma. If we choose as the domain for $\Psi(u, v)$ the domain $\mathscr{D} \equiv[\boldsymbol{C}-\{-\boldsymbol{J} / k\}] \times \boldsymbol{C}$, we see that $\Psi(u, v)$ satisfies the condition

$$
\operatorname{Re} \Psi(1,0) \equiv k+\lambda(J) \geqq k>0,
$$

because of (6), (8), and (12); moreover, 


$$
\begin{aligned}
\operatorname{Re} \Psi\left(i u_{2}, v_{1}\right) & =\frac{J\left(k v_{1}+\lambda J\right)+\lambda k^{2} u_{2}^{2}}{k^{2} u_{2}^{2}+J^{2}}, \quad \lambda \equiv \lambda(J), \\
& \leqq \frac{J(2 \lambda J-k)+k u_{2}^{2}(2 \lambda k-J)}{2\left(k^{2} u_{2}^{2}+J^{2}\right)}
\end{aligned}
$$

holds when $v_{1} \leqq-\left(1+u_{2}^{2}\right) / 2$. Hence $\Psi(u, v)$ satisfies condition (c) of Lemma A. $\operatorname{Re}\left[z f^{\prime}(z) / f(z)\right]>0$ in $U$ because $f \in S^{*}$, and this along with the hypotheses (1)-(8) imply that the right-hand side of (14) also has a positive real part in $U$. Hence $\Psi(u, v)$ satisfies all the hypotheses of Lemma $A$ in the domain $\mathscr{D}$ so that we can conclude that $P(z)$ satisfies the relation

$$
\operatorname{Re} P(z)=\operatorname{Re}[\beta p(z)+q(z)-J] \geqq 0
$$

in the disc $U$. If we take into account (5) and (10) along with (16), we conclude that

$$
\beta \operatorname{Re} \frac{z F^{\prime}(z)}{F(z)} \geqq J-\operatorname{Re} \frac{z \Phi^{\prime}(z)}{\Phi(z)}-\gamma \geqq 0
$$

holds in $U$. Hence $F \in S^{*}$.

It is worth noting that (17) gives us more than the conclusion $F \in S^{*}$; it gives us $F \in S^{*}(\varepsilon)$, where $\varepsilon=\inf \left[J-\gamma-\operatorname{Re}\left(z \Phi^{\prime}(z) / \Phi(z)\right]\right.$. Hence for appropriate choices of $J, \gamma, \Phi(z), F$ is more than "just" starlike. This will be pursued later on in the note.

Now we show the existence of a single-valued analytic function $F(z)$ that satisfies (9), given $\alpha, \beta, \gamma, \delta, \Phi(z), \varphi(z)$ and $f(z)$. That requirement is equivalent to showing the existence of a singlevalued analytic function $F(z)$ satisfying

$$
z^{\gamma} \Phi(z) F^{\beta}(z) \equiv(\beta+\gamma) \int_{0}^{z} f^{\alpha}(t) \varphi(t) t^{\hat{\delta}-1} d t
$$

in the disc $U$. The powers in (18) will be defined as our proof of the existence of $F(z)$ progresses.

Set $w(z)=[f(z) / z]^{\alpha} \equiv 1+\cdots$, which is a single-valued function analytic in $U$; moreover, $w(z) \neq 0$ there. Now set

$$
h(z) \equiv\left[z^{\delta} f^{\alpha}(z) \varphi(z)\right]^{1 /(\alpha+\delta)}=z[w(z)]^{1 /(\alpha+\delta)}=z \cdots,
$$

which is also a single-valued function analytic in $U$; moreover, $[h(z) / z] \neq 0$ there. Hence the function satisfies

$$
(\alpha+\delta) \frac{z h^{\prime}(z)}{h(z)}=\alpha z \frac{f^{\prime}(z)}{f(z)}+\delta+\frac{z \varphi^{\prime}(z)}{\varphi(z)} .
$$

But $f \in S^{*}$, and (19) along with (1), (3), and (7) imply $h \in S^{*}$. The function 


$$
g(z) \equiv \frac{\beta+\gamma}{z^{\beta+\gamma}} \int_{0}^{z} f^{\alpha}(t) \varphi(t) t^{\delta-1} d t=\frac{\beta+\gamma}{z^{\beta+\gamma}} \int_{0}^{z} \frac{h^{\alpha+\delta}(t)}{t} d t=1+\cdots
$$

is a single-valued function analytic in $U$. We wish to show that $g(z) \neq 0$ in $U$, and to do that we set $\tau=h(t)$ and $t=H(\tau)$, the inverse. If $L$ denotes the line segment joining $\tau=0$ to $\tau=h(z)$, then the inverse image of $L$ is a curve $l$ in the disc $U$; here we have made use of the fact that $L \subset h(U)$, i.e., $h(U)$ is a starlike domain. Hence

$$
g(z)=\frac{\beta+\gamma}{z^{\beta+\gamma}} \int_{l} \frac{h^{\alpha+\delta}(t) d t}{t}=\frac{\beta+\gamma}{z^{\beta+\gamma}} \int_{L} \frac{\tau^{\alpha+\delta} H^{\prime}(\tau) d \tau}{H(\tau)} .
$$

Now we set $\tau=\rho e^{i \theta}, R=|h(z)|, \theta$ fixed, in the last relation to obtain

$$
g(z) \equiv \frac{\beta+\gamma}{z^{\beta+\gamma}} \int_{0}^{R} \rho^{\alpha+\dot{o}-1} e^{i(\alpha+\delta) \theta}\left[\frac{\rho e^{i 0} H^{\prime}\left(\rho e^{i \theta}\right)}{H\left(\rho e^{i \theta}\right)}\right] d \rho,
$$

which implies

$$
|g(z)| \geqq \frac{\beta+\gamma}{|z|^{\beta+\gamma}} \int_{0}^{z} \rho^{\alpha+\delta-1} \operatorname{Re} \frac{\rho e^{i \theta} H^{\prime}\left(\rho e^{i \theta}\right)}{H\left(\rho e^{i \theta}\right)} d \rho .
$$

Since $h \in S^{*}$, in each closed disc in $U$ we can find positive constants $M$ and $N$ such that

$$
\operatorname{Re}\left[\frac{t h^{\prime}(t)}{h(t)}\right] \geqq M>0, \quad\left|\frac{t h^{\prime}(t)}{h(t)}\right| \leqq M
$$

in that closed disc; in particular these last relations hold for all $t \in l$, for some $M>0$ and $N>0$. Hence

$$
|g(z)| \geqq \frac{\beta+\gamma}{|z|^{\beta+\gamma}} \int_{0}^{R} \rho^{\alpha+\delta-1} \frac{M}{N^{2}} d \rho>0 .
$$

Hence $g(z) \neq 0$ in $U$.

We now introduce the function $g_{1}(z) \equiv g(z) / \Phi(z)$ which is analytic in the disc $U$ and satisfies $g_{1}(0)=1$ and $g_{1}(z) \neq 0$ there. Consequently we can define a single-valued function $F(z)$,

$$
F(z) \equiv z\left[g_{1}(z)\right]^{1 / \beta}=z+\cdots
$$

which is analytic in $U$ and satisfies the relations

$$
F^{\beta}(z) \equiv z^{\beta} g_{1}(z) \equiv \frac{z^{\beta} g(z)}{\Phi(z)} \equiv \frac{\beta+\gamma}{z^{\gamma} \Phi(z)} \int_{0}^{z} f^{\alpha}(t) \varphi(t) t^{\hat{\delta}-1} d t,
$$

which leads us to (9). The proof is now complete.

A weaker form of the theorem which is easier to apply is given by the following corollary. 
CoROLlaRY 1. Let $\Phi(z)=1+\cdots$ and $\varphi(z)=1+\cdots$ be analytic functions defined in the disc $U$, with $\varphi(z) \cdot \Phi(z) \neq 0$ there, and let $\alpha, \beta, \gamma, \delta$, and $J$ be real constants satisfying the conditions $\alpha>0$, $(\alpha+\delta)=(\beta+\gamma)$, and

$$
\delta+\operatorname{Re} \frac{z \Phi^{\prime}(z)}{\varphi(z)} \geqq J \geqq \gamma+\operatorname{Re} \frac{z \Phi^{\prime}(z)}{\Phi(z)}
$$

in $U$. Now if $f \in S^{*}$, then the function $F(z)$ defined in (9) is also in $S^{*}$.

The corollary follows directly from Theorem 1.

3. Integral operators that map $S^{*}$ into $S^{*}$. An operator defined on $S^{*}$, that maps $S^{*}$ into (or onto) $S^{*}$ is called a starlike operator. For example, the operator $\mathscr{I}$ defined by (9) is a starlike (integral) operator. In this section we shall study several integral operators, which are special cases of (9), to show the power of our method. As noted earlier, our results are considerably sharper than those obtained by other authors up to this time.

THEOREM 2. If $\beta>0, \gamma \geqq 0$ and $f \in S^{*}$, then the function $F(z)$ defined by

$$
F(z) \equiv\left[\frac{\beta+\gamma}{z^{\gamma}} \int_{0}^{z} f^{\beta}(z) t^{\gamma-1} d t\right]^{1 / \beta}=z+\cdots
$$

is again an element of $S^{*}$.

Proof. If we choose $\alpha=\beta, \gamma=\delta, \phi(t) \equiv \Phi(z) \equiv 1$, and $J=\gamma$, then (23) is satisfied, and hence, by Corollary $1, F \in S^{*}$.

The preceding result is due to R. Singh [7] for $\beta$ and $\gamma$ positive integers.

If we use Theorem 1 and the useful relations (10), (13), and (17), then we can obtain somewhat more to demonstrate the idea in hand, and to avoid complicated computations-because our results are not the most precise-we develop certain special cases already in the literature.

(A) Let $\beta=1$ in (24), and $0 \leqq \gamma \leqq 1$. Then it is easy to verify that the constant

$$
J_{1} \equiv\left[\frac{2 \gamma-1+\sqrt{(2 \gamma-1)^{2}+8(1+\gamma)}}{4}\right]
$$

has the following properties. (i) $1+\gamma>J_{1} \geqq 1+\gamma / 2 \geqq \gamma$, $J_{1}-\lambda\left(J_{1}\right) \geqq 0$, where $\lambda\left(J_{1}\right)=J_{1} / 2\left[\left(1+\gamma-J_{1}\right)\right]$, and (iii) $\gamma \geqq J_{1}-\lambda\left(J_{1}\right)$. 
Hence we can use Theorem 1, with $J=J_{1}$, to obtain (16) in the form

$$
\operatorname{Re} P(z)=\operatorname{Re}\left[\frac{z F^{\prime}(z)}{F(z)}+\gamma-J_{1}\right] \geqq 0,
$$

which yields

$$
\operatorname{Re} \frac{z F^{\prime}(z)}{F(z)} \geqq J_{2} \equiv \frac{-(2 \gamma+1)+\sqrt{(2 \gamma-1)^{2}+8(1+\gamma)}}{4}, \quad 0 \leqq \gamma \leqq 1 .
$$

The relation (25) implies that $F \in S^{*}\left(J_{2}\right)$. In particular, for $\gamma=0$, $F \in S^{*}(1 / 2)$, which is a well-known property of convex $F$; for $\gamma=1$, we have $F \in S^{*}(\sqrt{17}-3) / 4$. This very last result is an improvement of an earlier one due to Libera, who showed $F \in S^{*}$.

(B) If we choose the same $\beta, J_{1}$ and $\lambda\left(J_{1}\right)$ as in (A) above, with $\gamma \geqq 1$, then we can conclude that the transform $F$ satisfies (25), which is an improvement of a result due to Bernardi [1], who showed that $F \in S^{*}$ for $\gamma=1,2,3, \cdots$.

In a similar manner, we can obtain similar extensions of $R$. Singh's generalization of Bernardi's result [7]. Since the order of starlikeness of the transform $F$ is not precise, we do not present the details here.

It is worth noting that the transforms of the form (24), for $f \in S^{*}$, represent generalizations of the alpha-convex functions introduced by Mocanu [6]; these functions arise with the choices $\beta=1 / \alpha, \gamma=0$. However, our technique does not yield new results for those functions.

Theorem 3. If $0 \leqq \alpha \leqq 1, \alpha \leqq \beta$, and if $f \in S^{*}$, then the function

$$
F(z) \equiv\left[z^{\beta-1} \int_{0}^{z}\left(\frac{f(t)}{t}\right)^{\alpha} d t\right]^{1 / \beta}=z+\cdots
$$

is again an element of $S^{*}$.

Proof. A proof can be given that appeals to Corollary 1, with $\alpha+\delta=\beta+\gamma=1, \varphi(z) \equiv \Phi(z) \equiv 1, \gamma \leqq J \leqq \delta$, where $J=1-\alpha \geqq 0$.

As in the illustrative examples (A) and (B) above, it is possible to obtain sharper results by examining (17) in each case that Theorem 1 is used. For example, the following example illustrates the idea, used in (A) and (B) above, and it provides an extension of a result due to Merkes and Wright [5].

(C) If $0 \leqq \alpha \leqq 1$, and if $f \in S^{*}$, then the function 


$$
F(z) \equiv \int_{0}^{z}\left(\frac{f(t)}{t}\right)^{\alpha} d t
$$

belongs to $S^{*}\left(J_{3}\right)$, where

$$
J_{3} \equiv \frac{1}{4}\left[(1-2 \alpha)+\sqrt{4 \alpha^{2}-4 \alpha+9}\right] .
$$

To obtain that last result, we can uso Theorem 1 with $\gamma=0$, $\alpha+\delta=\beta=1, \delta \geqq 0,1 / 2 \leqq J_{3} \leqq 1, J_{3}-\lambda\left(J_{3}\right) \leqq \delta$ and $J_{3}$ given by (28), to show that (13) yields

$$
\operatorname{Re} \frac{z F^{\prime}(z)}{F(z)} \geqq J_{3} \equiv \frac{(1-2 \alpha)+\sqrt{4 \alpha^{2}-4 \alpha+9}}{4} .
$$

Merkes and Wright showed that $f \in S^{*}$ implies $F \in K, 0 \leqq \alpha \leqq 1$, and consequently $f \in S^{*}(1 / 2)$, which is valid for all $f \in K$. Our result is more precise, though not the best one.

We conclude this section with a simple theorem that yields expressions for starlike functions of curious forms.

THEOREM 4. If $\alpha>0, \eta \geqq 0, \gamma+\eta \geqq 0$, and if $f \in S^{*}$, then the function

$$
F(z) \equiv\left[\frac{\alpha+\gamma+\eta}{z^{\gamma}} \int_{0}^{z} f^{\alpha}(t) t^{\gamma+\eta-1} d t\right]^{1 /(\alpha+\eta)}=z+\cdots
$$

is also starlike in $U$.

For $\gamma+\eta=1, \alpha=1, \eta=0,1,2, \cdots$, we obtain the sequence

$$
\left[2 z^{n-1} \int_{0}^{z} f(t) d t\right]^{1 /(n+1)}=z+\cdots, \quad n=0,1,2, \cdots,
$$

and for $\gamma=0, \alpha=1, \eta=0,1,2, \cdots$, we obtain the sequence

$$
\left[(n+1) \int_{0}^{z} f(t) t^{n-1} d t\right]^{1 /(n+1)}=z+\cdots, \quad n=0,1,2, \cdots,
$$

of elements of $S^{*}$.

4. Integral operators the map $K$ into $S^{*}$ and/or $K$.

THEOREM 5. Let $\alpha, \beta, \gamma, \delta, J, \lambda(J), \varphi(z)$, and $\Phi(z)$ satisfy the hypothesis of Theorem 1, except that (7) is replaced by the condition

$$
\frac{\alpha}{2}+\delta+\operatorname{Re} \frac{z \varphi^{\prime}(z)}{\varphi(z)} \geqq \max [0, J-\lambda(J)] .
$$

If $f \in K$, then $F \in S^{*}$, where $F(z)$ is defined by (9). 
A proof of Theorem 5 can be given that parallels the proof of Theorem 1. It is worth noting that in such a proof, we use the fact that $f \in S^{*}(1 / 2)$, which follows from the hypothesis that $f \in K$. Hence the conclusion of Theorem 5 is valid for all $f \in S^{*}(1 / 2)$.

It is possible to imitate examples (A), (B), and (C) above to show that if $f \in K$ (or if $f \in S^{*}(1 / 2)$ ) then the transform of $f$ is smoother than $f$, for some transforms. For example,

(D) if $\gamma>0, f \in K$, then the function

$$
F(z) \equiv \frac{1+\gamma}{z^{\gamma}} \int_{0}^{z} f(t) t^{\gamma-1} d t
$$

is an element of $K\left(J_{2}\right)$, where $J_{2}$ is given by (25), that is $F$ is convex of order $J_{2}$. To prove this result, we employ a partial integration to obtain

$$
z F^{\prime}(z) \equiv \frac{1+\gamma}{z^{\gamma}} \int_{0}^{z}\left[t f^{\prime}(t)\right] t^{r-1} d t
$$

For $f \in K,\left(z f^{\prime}\right) \in S^{*}$, so that we can use Theorem 2 , with $\beta=1$, to conclude $\left(z F^{\prime}\right) \in S^{*}$. By using examples (A) and (B) above we conclude that $\left(z F^{\prime}\right) \in S^{*}\left(J_{2}\right)$ and hence $F \in K\left(J_{2}\right)$. This last is an improvement of an earlier result due to Bernardi [1], who proved that $f \in K$ implies $F \in K$.

In a similar way we can show that for $0 \leqq \alpha \leqq 1, f \in K$, the function

$$
F(z) \equiv \int_{0}^{z}\left(\frac{f(t)}{t}\right)^{\alpha} d t
$$

is an element of $K(1-\alpha / 2)$, which is an improvement over the Merkes-Wright result that $F \in K(1-\alpha)$ [5].

5. Integral operators that map $S^{*} \times K$ into $S^{*}$. A modification of formula (9) gives us a very general transformation and the following result.

THEOREM 6. Let $\alpha, \beta, \gamma, \delta$, and $\rho$ be real constants satisfying the conditions, $\alpha \geqq 0, \beta>0, \alpha+\delta=\beta+\delta>0$ and

$$
0 \leqq \frac{1}{2} \rho \leqq \begin{cases}\delta, & \gamma \leqq 0 \\ \min \left[\delta, \delta-\gamma+\frac{1}{2} \min \left(\frac{\beta}{\gamma}, \frac{\gamma}{\beta}\right)\right], & \gamma>0 .\end{cases}
$$

If $f \in S^{*}, g \in K$, then the function 


$$
F(z) \equiv\left[\frac{\beta+\gamma}{z^{\gamma}} \int_{0}^{z} f^{\alpha}(t) g^{\rho}(t) t^{\delta-\rho-1} d t\right]^{1 / \beta}=z+\cdots
$$

is a starlike function.

Proof. We can use Theorem 1, with $\varphi(z) \equiv[g(z) / z]^{\circ}, \Phi(z) \equiv 1$, and the relation

$$
\operatorname{Re} \frac{z \varphi^{\prime}(z)}{\varphi(z)}=\rho \operatorname{Re}\left[\frac{z g^{\prime}(z)}{g(z)}-1\right] \geqq-\frac{1}{2} \rho
$$

to complete a proof.

The following result contains recent ones due to Causey and White [2].

CoROLlaRy 2. Let $\alpha, \beta, \gamma, \delta$, and $\rho$ be real constants such that $\alpha>0, \alpha+\delta=\beta+\gamma, 0 \leqq \rho / 2 \leqq \min [\delta, \delta-\gamma]$. If $f \in S^{*}, g \in K$, then $F(z)$ defined by (30) is a starlike function.

Causey and White obtained this last result, but for $\beta=1,2, \cdots$ and $(\beta+\gamma)=1,2, \cdots$. However, our method shows more, at least for $\beta=1$, in which case the transform $F(z)$ is smoother that $f(z)-$ as in examples (A), (B), and (C) above.

It is worth noting that Theorem 6 must be used because Corollary 2 may not be applicable. For example, if $f \in S^{*}$, if $g \in K$, then the function

$$
F(z) \equiv \frac{2}{z} \int_{0}^{z}\left(\frac{f(t)}{t}\right)^{5 / 4}\left(\frac{g(t)}{t}\right)^{1 / 2} d t
$$

is starlike. Corollary 2 cannot be used here.

Another transformation of the form (30) that appears promising is that given by

$$
F(z) \equiv \frac{2}{z} \int_{0}^{z} \frac{f(t) g(t)}{t} d t
$$

which suggests that transformations of the form

$$
F(z) \equiv \frac{2}{z} \int_{0}^{z} f(t)\left(h^{\prime}(t)\right)^{\alpha} d t,
$$

where $\alpha$ is real and $h(t) \in S^{*}$, may yield interesting results. We shall pursue that elsewhere.

It is important to note that the results of this section remain valid if the "weight function" $g$ is an element of $S^{*}(1 / 2)$; all our proofs used only that property of convex $g(z)$. 
6. Integral operators that map $K \times K$ into $S^{*}$. In this section we describe integral operators that transform the set $K \times K$ into $S^{*}$. There are no exciting results here, but we believe some of the ideas introduced here are worthy of at least a superficial development.

THEOREM 7. Let $\alpha, \beta, \gamma, \delta$, and $\rho$ be real constants such that $\alpha \geqq 0, \beta>0, \alpha+\delta=\beta+\gamma>0$ and

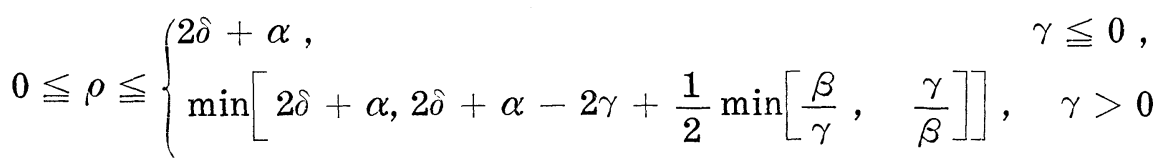

hold. If $f \in K, g \in K$, then the function $F(z)$, defined in (30) is a starlike function.

A proof of this result could be given, one that looks like all the others in this note. We do remark that our result is a little more general than a similar one due to Causey and White [2] who studied the transform

$$
F(z) \equiv\left[\frac{\alpha+\gamma}{z^{\gamma}} \int_{0}^{z} f^{\alpha}(t) g^{\gamma-1}(t) d t\right]^{1 / \alpha}=z+\cdots
$$

for $f \in K$ and $g \in K$. However the transform

$$
\mathscr{F}(f) \equiv\left[\frac{8}{3 z^{2}} \int_{0}^{z} f^{2 / 3}(t) g(t) d t\right]^{3 / 2}=z+\cdots
$$

can be studied by our methods and not by the methods used by Causey and White. Indeed, they considered only the values $\alpha, \gamma=$ $1,2, \cdots$, with $\gamma \leqq 1+\alpha$ in (31).

Again, it is important to note that we use only the property that $f \in S^{*}(1 / 2)$ and $g \in S^{*}(1 / 2)$, for convex $f$ and $g$, to obtain our conclusion. Hence it is clear that sharper results must exist for transforms (30) defined on $K \times K$.

7. Conclusion. We have outlined a method that permits us to obtain reasonably sharp results concerning (starlike) integral transformations of the form (9) defined on $S^{*}, K, S^{*} \times K$, and $K \times K$. We have shown that such transforms sometimes map elements of the pre-image space onto "smoother" elements in the image space. It would be of some interest to obtain the exact order of smoothness of the image function $F(z)$ when compared to the pre-image function $f(z)$. 


\section{REFERENCES}

1. S. D. Bernardi, Convex and starlike univalent functions, Trans. Amer. Math. Soc., 135 (1969), 429-446.

2. W. M. Causey and W. L. White, Starlikeness of certain functions with integral representations, J.A.M.A., 64 (1978), 458-466.

3. Z. Lewandowski, S. Miller, and E. Zlotkiewicz, Generating functions for some classes of univalent functions, Proc. Amer. Math. Soc., 56 (1976), 111-117.

4. R. J. Libera, Some classes of regular univalent functions, Proc. Amer. Math. Soc., 16 (1965), 755-758.

5. E. P. Merkes, and D. J. Wright, On the univalence of a certain integral, Proc. Amer. Math. Soc., 27 (1971), 97-100.

6. S. Miller, P. Mocanu and M. Reade, Bazilevic functions and generalized convexity, Rev. Roum. Math. Pures et Appl. XIX, No. 2 (1974), 213-224.

7. R. Singh, On Bazilevic functions, Proc. Amer. Math. Soc., 38 (1973), 261-271.

Received January 21, 1978. The first and third authors work was begun while these authors were USA-Romania Exchange Scholars.

State University of New York, Brockport, NY 14420

Babes-Bolyai University, 3400 Cluj-Napoca, Romania

AND

University of Michigan, ANn ARbor, MI 48109 


\section{PACIFIC JOURNAL OF MATHEMATICS}

EDITORS

RICHARD ARENS (Managing Editor)

University of California

Los Angeles, California 90024

C. W. Curtis

University of Oregon

Eugene, OR 97403

C. C. MOORE

University of California

Berkeley, CA 94720
J. DUGUNDJI

Department of Mathematics University of Southern Californı Los Angeles, California 90007

R. Finn and J. Milgram Stanford University Stanford, California 94305

\section{ASSOCIATE EDITORS}

E. F. BECKENBACH

B. H. NEUMANN

F. WOLF

K. YoSHIDA

\section{SUPPORTING INSTITUTIONS}

UNIVERSITY OF BRITISH COLUMBIA
CALIFORNIA INSTITUTE OF TECHNOLOGY
UNIVERSITY OF CALIFORNIA
MONTANA STATE UNIVERSITY
UNIVERSITY OF NEVADA, RENO
NEW MEXICO STATE UNIVERSITY
OREGON STATE UNIVERSITY
UNIVERSITY OF OREGON

UNIVERSITY OF SOUTHERNEALIFORNIA STANFORD UNIVERSITY UNIVERSITY OF HAWAII UNIVERSITY OF TOKYO UNIVERSITY OF UTAH WASHINGTON STATE UNIVERSITY UNIVERSITY OF WASHINGTON 


\section{Pacific Journal of Mathematics \\ Vol. 79 , No. 1 \\ May, 1978}

Teófilo Abuabara, A remark on infinitely nuclearly differentiable

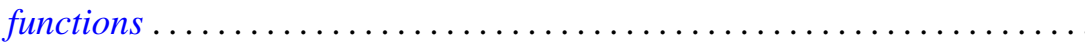

David Fenimore Anderson, Projective modules over subrings of $k[X, Y]$

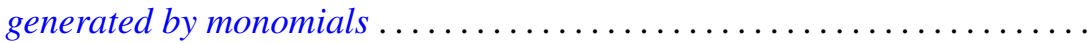

Joseph Barback and Thomas Graham McLaughlin, On the intersection of

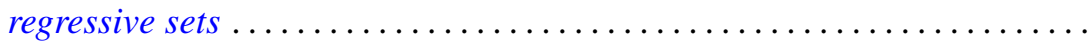

Murray Bell, John Norman Ginsburg and R. Grant Woods, Cardinal inequalities for topological spaces involving the weak Lindelof

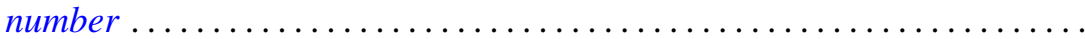

Laurence Richard Boxer, The space of ANRs of a closed surface ............

Zvonko Cerin, Homotopy properties of locally compact spaces at

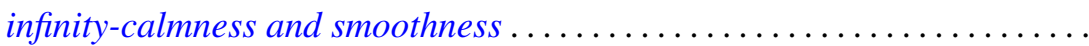

Isidor Fleischer and Ivo G. Rosenberg, The Galois connection between partial functions and relations..................................

John R. Giles, David Allan Gregory and Brailey Sims, Geometrical implications of upper semi-continuity of the duality mapping on a Banach

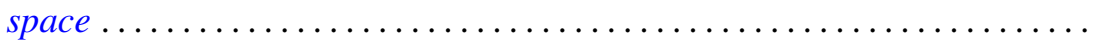

Troy Lee Hicks, Fixed-point theorems in locally convex spaces ............ Hugo Junghenn, Almost periodic functions on semidirect products of transformation semigroups ........................

Victor Kaftal, On the theory of compact operators in von Neumann algebras. II . . . .

Haynes Miller, A spectral sequence for the homology of an infinite

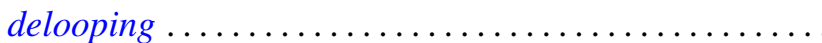

Sanford S. Miller, Petru T. Mocanu and Maxwell O. Reade, Starlike integral operators...

Stanley Stephen Page, Regular FPF rings ...............

Ghan Shyam Pandey, Multipliers for C, 1 summability of Fourier series ...

Shigeo Segawa, Bounded analytic functions on unbounded covering surfaces...

Steven Eugene Shreve, Probability measures and the C-sets of

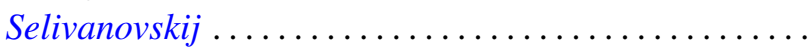

Tor Skjelbred, Combinatorial geometry and actions of compact Lie groups....

Alan Sloan, A note on exponentials of distributions.

Colin Eric Sutherland, Type analysis of the regular representation of a nonunimodular group.

Mark Phillip Thomas, Algebra homomorphisms and the functional

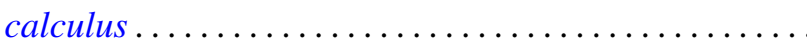

Sergio Eduardo Zarantonello, A representation of $H^{p}$-functions with

$0<p<\infty$. 